\title{
Pore dynamics of osmotically stressed vesicles
}

\author{
Yan Levin and Marco A. Idiart \\ Instituto de Física, UFRGS, Caixa Postal 15051, CEP 91501-970, Porto Alegre, RS, Brazil
}

(Dated: November 13, 2018)

\begin{abstract}
We present a theory for pore dynamics of osmotically stressed vesicles. When a liposome with an internal concentration of solute is placed inside a solute-depleted medium, an osmotic flow of solvent through the lipid bilayer leads to swelling of vesicle and to increase in membrane surface tension. This can result in membrane rupture and opening of thermal pores. Depending on the internal concentration of solute and the size of the vesicle, pores can close rapidly or be long-lived. We find that the life span of the long-lived pores scales non-trivially with the size of the liposome. Closure of the long-lived pore is followed by a rapid flicker-like opening and closing of short-lived pores. Our model is consistent with the observation of long-lived pores in red blood cell ghosts.
\end{abstract}

Opening of stable pores in cellular phospholipid membranes is an important step for drug delivery and gene therapy. A number of methods have been explored to this end. Electroporation [1] is the method of choice when gene delivery is performed in vitro. It is, however, associated with a significant cell mortality limiting its practical use. Holes in cellular membranes have also been opened by intense illumination 2, 3]. The mechanism leading to pore formation under these circumstances is still not fully understood. It, however, appears to be similar to the action of laser tweezers [4], a part of the low dielectric constant lipid membrane is sucked into the laser trap, stretching the membrane until rupture.

For drug delivery the goal is to introduce a controlled amount of substance to the specific disease site. The delivery system should minimize the potential side-effects by selectively targeting the affected region of the organism [5]. Liposomes can be made to contain drugs or genes and can be introduced into blood stream without provoking an immune system response. Liposomal affinity for specific tissue can be increased by varying the membrane lipidic composition or by including ligands that recognize and bind to specific receptors [6]. One example of this is the drug called Doxirubicin used in cancer chemotherapy. The liposomal encapsulation of Doxirubicin has been shown to efficiently deliver it to tumors, while minimizing the side effects, such as damage to heart muscle tissue.

When liposome ruptures, its content leaks out. It is precisely the dynamics of this rupture that is the subject of the present Letter. In particular, our goal is to understand the mechanism of pore opening and closure in the osmotically stressed vesicles. The situation which we attempt to model is the following. Liposomes are prepared inside a solution containing small molecules impermeable to lipid membrane at concentration $c_{0}$. They are then placed into a purified aqueous solution. The imbalance in solute concentration inside and outside the vesicles leads to osmotic flow of solvent through the semipermeable lipid membrane, resulting in swelling of vesicles and stretching of their membranes. As the tension increases, the energy barrier to pore nucleation decreases [7]. If there is enough solute inside the liposome, the barrier height eventually becomes comparable to the energy of thermal fluctuations. When this happens, membrane raptures and a pore opens [8]. One interesting question is what is the life span of this pore? Specifically, as a pore opens it relaxes the membrane tension. However, opening of a pore exposes the hydrophobic interior of membrane to water, leading to pore line (edge) tension. This hydrophobic effect induces a force which tries to re-seal the pore. Furthermore, a hole in membrane results in efflux of both solute and solvent, reducing the vesicle volume and surface tension. All these effects lead to an intricate interplay of time and length scales which control the pore closure.

We shall designate the radius of a vesicle as $R$ and the radius of a pore as $r$. The conservation of mass then leads to

$$
4 \pi \rho R^{2} \frac{d R}{d t}=j_{w}-\pi r^{2} \rho v,
$$

where $\rho$ is the density of water, $j_{w}$ is the osmotic flux and $v$ is the leak-out velocity. The leak-out velocity 9,10 , 11, 12] is determined by the balance between the shear stress, proportional to $\eta v / r$, and the osmotic pressure inside the vesicle $\Delta p$,

$$
v=\frac{\Delta p r}{3 \pi \eta},
$$

where $\eta$ is the solvent viscosity. Inside a swollen vesicle the osmotic pressure is compensated by the Laplace pressure, so that

$$
\Delta p=\frac{2 \sigma}{R},
$$

where $\sigma$ is the tension of a stretched membrane. In this work we shall concentrate on vesicles with fairly large internal concentrations of solute, close to $1 \mathrm{M}$. This corresponds to osmotic pressures as high as 20 atmospheres. Of course, before these extreme pressures can be built, the lipid membrane will rapture, leaking out some of the internal content and releasing the surface stress. At these high pressures, the thermal undulations of membrane can be ignored, and the membrane tension is controlled by the stretching modulus [13, 14, 15] $K_{s}$. The membrane elastic energy takes a Hook-like form

$$
E_{s}=\frac{1}{2} K_{s}\left(A-A_{0}\right)^{2}
$$


where $A_{0}=4 \pi R_{0}^{2}$ is the equilibrium surface area of the unstretched membrane. As the membrane is stretched beyond the elastic limit, pores are nucleated reducing the membrane tension. The elastic energy of a membrane with a pore is

$$
E(R, r)=\frac{1}{2} K_{s}\left[4 \pi\left(R^{2}-R_{0}^{2}\right)-\pi r^{2}\right]^{2}+2 \pi \gamma r,
$$

where $r$ is the pore radius and $\gamma$ is the pore line tension. The membrane surface tension is,

$$
\sigma(R, r)=\frac{\partial E_{s}}{\partial A}=K_{s}\left(4 \pi\left(R^{2}-R_{0}^{2}\right)-\pi r^{2}\right) .
$$

The osmotic current $j_{w}$ is determined by the membrane permeability $P$, the concentration difference of solute inside and outside the vesicle, and the Laplace pressure. A simple phenomenological expression for the osmotic current of water into the vesicle can be written as,

$$
j_{w}=P\left(4 \pi R^{2}-\pi r^{2}\right)\left[c-\frac{2 \sigma}{10^{3} k_{B} T N_{A} R}\right],
$$

where $k_{B}$ is the Boltzmann constant and $N_{A}$ is the Avogadro number. If the difference of molar $(M)$ solute concentration $c$, inside and outside the vesicle is not very large, the integrity of membrane will not be compromised, and a stationary state with $j_{w}=0$ will be achieved. Under these conditions the osmotic pressure is completely compensated by the Laplace pressure, resulting in zero net flux of solvent. For sufficiently large internal concentration $c_{0}$, a stationary state will not be achieved before membrane ruptures. It is precisely in this regime that we expect to see some interesting physics.

The growth of a pore is controlled by the rate at which the elastic energy is dissipated by the membrane viscosity $\eta_{m}$ [16],

$$
\eta_{m} l \frac{d r}{d t}=\sigma(R, r) r-\gamma,
$$

where $l$ is the membrane width. Since the membrane is impermeable to solute particles the internal solute concentration is modified only through the osmotic influx of solvent and the efflux of solute through the open pore, after membrane has ruptured. The continuity equation expressing this is

$$
\frac{4 \pi}{3} R^{3} \frac{d c}{d t}=-4 \pi R^{2} c \frac{d R}{d t}-\pi r^{2} c v,
$$

where we have assumed that solute is uniformly distributed inside the vesicle.

We are now in position to study the evolutionary dynamics of an osmotically stressed vesicle. The characteristic values of the parameters appearing in the above equations are: $4 \pi R_{0}^{2} K_{s}=0.2 \mathrm{~J} / \mathrm{m}^{2}, l=3.5 \mathrm{~nm}$, $\gamma=10^{-12} \mathrm{~J} / \mathrm{m}, \eta_{m}=100$ Pas,$\eta_{w}=10^{-3}$ Pas and $P=1.8 \times 10^{-4} \mathrm{~kg} / \mathrm{m}^{2} \mathrm{~s} M$.

The energy necessary to open a pore or radius $r$ is $\Delta E(R, r)=E(R, r)-E(R, 0)$. The form of $\Delta E(R, r)$

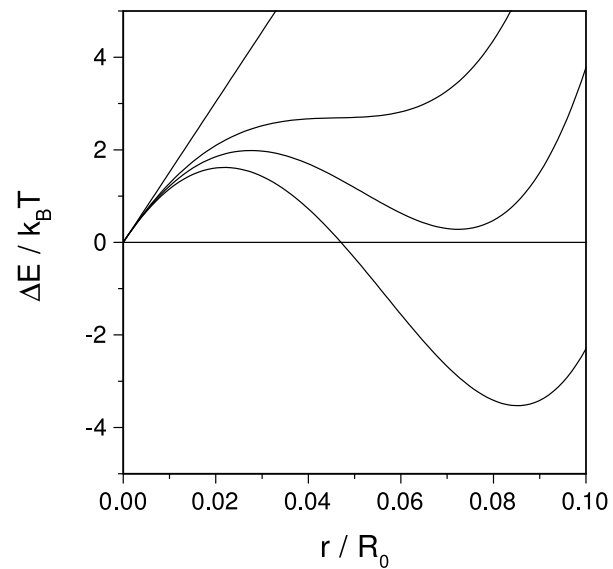

FIG. 1: Energy $\Delta E(R, r)$ necessary to open a pore of radius $r$ in a liposome with $R_{0}=100 \mathrm{~nm}$. The curves are, from top down, $R / R_{0}=1.0, R / R_{0}=1.0008, R / R_{0}=1.001$, and $R / R_{0}=1.0012$

as a function of pore size is plotted in Fig. 1 for various values of $R$. As the osmotic flow of solvent stretches the membrane, a minimum in $\Delta E(R, r)$ for $r \neq 0$ indicates energetic favorability of pore formation. However, to reach this minimum a sufficiently large thermal fluctuation is necessary to overcome the energy barrier. The probability of such a fluctuation occurring is proportional to the Boltzmann factor

$$
P(r) \sim e^{-\beta \Delta E\left(R, r^{*}\right)},
$$

where $\beta=1 / k_{B} T$ and $r^{*}$ is the critical pore size. Pores with $r<r^{*}$ will shrink and close while pores with $r \geq r^{*}$ will grow. The waiting time for appearance of a thermal fluctuation with enough energy to open a pore of radius $r^{*}$ is very long, unless $\Delta E\left(R, r^{*}\right) \approx k_{B} T$.

In the limit of large surface tensions the critical pore size is $r^{*} \approx \gamma / \sigma$ and the barrier height is

$$
\beta \Delta E_{b} \approx \frac{\pi \gamma^{2}}{\sigma k_{B} T} .
$$

We note that when membrane tension reaches $\sigma_{c}=$ $10^{-3} \mathrm{~J} / \mathrm{m}^{2}$, the barrier height is approximately $k_{B} T$. This value of $\sigma_{c}$ is in agreement with the tensions found to be necessary to rupture a mechanically stretched membrane [17, 18]. The minimum solute concentration necessary for a pore to open is

$$
c_{0}^{\min } \approx \frac{2 \sigma_{c}}{10^{3} k_{B} T N_{A} R_{0}},
$$

which for a vesicle of $100 \mathrm{~nm}$ is approximately $10 \mathrm{mM}$.

The dynamics then proceeds as follows: Eqs. (11), (8), and (9) are solved numerically, using Euler's method with a stepsize of $\delta t=0.1 \mu \mathrm{s}$, to find the evolution of $R(t)$, $r(t)$, and $c(t)$. When the surface tension becomes such that, $\Delta E_{b} \approx k_{B} T$, a pore of size $r^{*}$ opens, see Fig. 2, 
and the internal content of the liposome begins to leakout. This process reduces the volume of the vesicle and decreases the membrane tension, until the pore closes. Once this happens, the osmotic swelling of the liposome re-starts. As the energy barrier to pore nucleation drops down to $k_{B} T$ a new pore is opened, etc. The process stops when the internal solute concentration reaches $c_{0}^{\min }$ and a stationary state with $j_{w}=0$ is established.

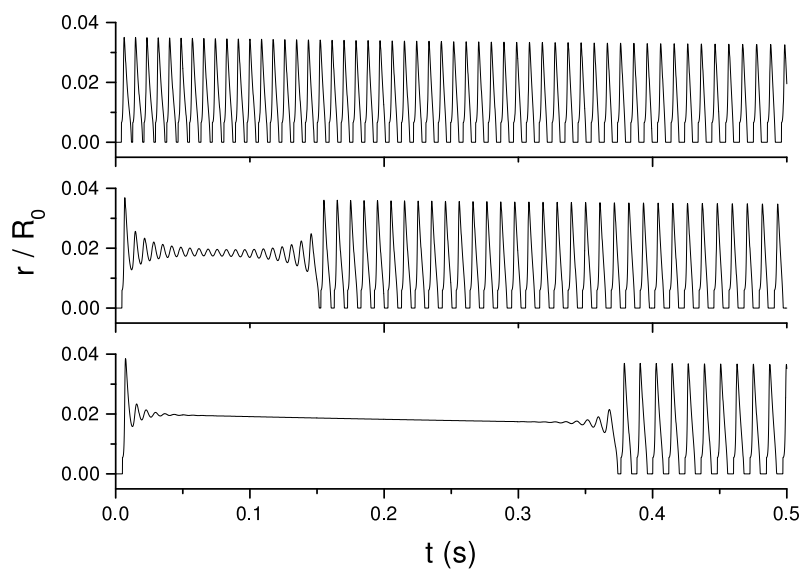

FIG. 2: Radius of a pore as a function of time for vesicles of $R_{0}=200 \mathrm{~nm}$ (topmost), $220 \mathrm{~nm}$ and $240 \mathrm{~nm}$ (bottommost), for initial concentration $c_{0}=0.5 \mathrm{M}$. Note that for vesicle with $R_{0}=200 \mathrm{~nm}$ the pores are short-lived, while for larger vesicles, long-lived pore opens first.

For small vesicles, pores open and close very quickly with the characteristic time $\tau_{f} \sim 10^{-2}$, resulting in a periodic flickering. For liposomes with $R_{0}>R_{0}^{c}\left(c_{0}\right)$ a longlived pore appears, see Fig. 3 . The critical size of a liposome $R_{0}^{c}\left(c_{0}\right)$, necessary for nucleating a long-lived pore depends on the internal solute concentration. Larger the initial solute concentration $c_{0}$, smaller will be the size of the vesicle which supports a long-lived pore. The long life span of these pores is the result of a "wash-out" effect in which the osmotic flux is almost completely compensated by the leak-out rate of solute through the pore. When solute concentration inside the vesicle drops below the critical value, $c_{c}\left(R_{0}\right)$, the long-lived pore closes. This value is insensitive to the initial solute concentration $c_{0}$, but depends strongly on the vesicle size $R_{0}$. The life span of a long-lived pore $\tau$ scales with the vesicle size $R_{0}$,

$$
\tau \sim R_{0}^{\nu},
$$

with $\nu \approx 2.3-2.4$, see Fig. 3. After the long-lived pore has closed, it is followed by a sequence of short-lived pores with the characteristic life span $\tau_{f}$.

We have checked that the pore dynamics is not sensitive to the specific mechanism of pore opening. In particular, even if the pores are opened stochastically, with the probability given by the Boltzmann distribution, the rate of leakage and the life span of the long-lived pores is affected very little.

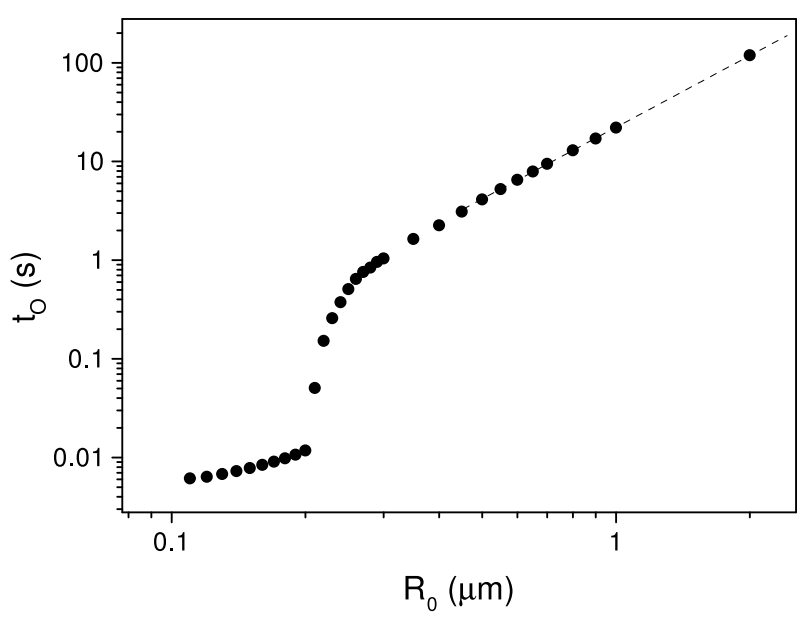

FIG. 3: Life span of the first-open pore as a function as a function of vesicle size $R_{0}$ for $c_{0}=0.5 \mathrm{M}$. Note the appearance of the critical vesicle size $R_{0}^{c}\left(c_{0}\right)$ which can sustain long-lived pores.

Up to now we have not taken into account the diffusive efflux of solute through the open pores. The diffusive current through a pore of size $r$ is approximately

$$
j_{D} \sim \pi r^{2} c \frac{D}{R_{0}} .
$$

This leads to the decline of internal solute concentration governed by the conservation equation,

$$
\frac{4 \pi}{3} R_{0}^{3} \frac{d c}{d t}=-j_{D},
$$

the solution of which is

$$
c(t)=c_{0} e^{-\frac{t}{\tau_{e}}},
$$

where the effusion time $\tau_{e}$ is

$$
\tau_{e}=\frac{4 R_{0}^{4}}{3 r^{2} D} .
$$

Using $D \approx 10^{-9} \mathrm{~m}^{2} / \mathrm{s}$, appropriate for small organic molecules such as sucrose, and $r=r^{*} \approx \gamma / \sigma_{c} \approx 1 \mathrm{~nm}$, we see that for liposomes with $R_{0}=50 \mathrm{~nm}$, the time for effusion is $\tau_{e} \approx 10^{-2} \mathrm{~s}$. This is comparable to the flicker time $\tau_{f}$. Therefore, for small vesicles effusion is an important mechanism for the loss of solute. On the other hand, for liposomes with $R_{0}=100 \mathrm{~nm}$ and above, effusion is only marginally relevant.

Long lived pores have been observed in red blood cell ghosts [19, 20], their size dependent on the ionic strength of the surrounding medium [21]. No theory, up to date, was able to account for these long-lived pores. Holes were predicted to either grow indefinitely, which would result in ghost vesiculation, or to close completely [22]. Our model provides a dynamical mechanism for pore stabilization, consistent with the experimental observations. 
In the specific case of red blood cell ghosts the ratio $\gamma / K_{s}$ has to be ajusted to account for the large radius $r / R_{0}$ observed in these experiments [19, 20].

In aqueous solutions the phospholipid membranes acquire a net negative charge. At physiological concentrations, $154 \mathrm{mM}$ of $\mathrm{NaCl}$, the Debye length, however, is quite short, less then $1 \mathrm{~nm}$ and the electrostatic interactions are strongly screened [23]. We, therefore, do not expect that electrostatics will significantly modify the basic conclusions of our theory, beyond the renormalization of membrane line 22] and surface tension. However, further, investigations in this direction are necessary and will be the subject of future work.

Finally, it is curious to note a strong similarity between the rupture of osmotically stressed liposomes and bursting of Hydra cells aggregates 24]. Hydra and marine sponges can generate functional organisms from random cell aggregates purely through the intercellular interaction. This morphogenesis is characterized by the cavity formation followed by swelling and violent bursting, which expels the internal fluid and dead cells, see Fig. 5 .

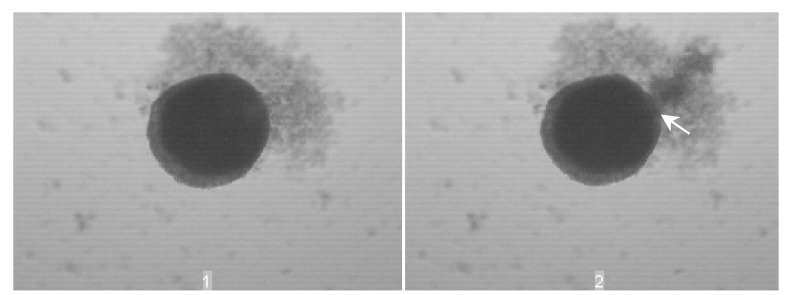

FIG. 4: A sequence of bursts in hydra aggregates. Picture one shows an aggregate with a diffuse cloud of expelled cells from the previous explosions, while picture two shows a cellular aggregate in the process of bursting. Arrow indicates the site of the burst.

Unlike the liposomes, cellular aggregates are too massive for temperature to be of any relevance. Instead the cellular Brownian motion is driven by the metabolic fluctuations of the cytoskeleton [25]. We can, therefore, expect that a theory similar to the one presented above for the liposomes, might also apply to osmotically driven bursting of cellular aggregates.

The authors are grateful to Ms. V. A. Grieneisen for kindly providing the photos of bursts in hydra aggregates, Fig. 4. This work was supported in part by the Brazilian agencies CNPq and FAPERGS.
[1] R. Benz, F. Beckers, and U. Zimmermann, J. Membr. Bio. 48, 181 (1979).

[2] R. Bar-Ziv, T. Frisch, and E. Moses, Phys. Rev. Lett. 75, 3481 (1995).

[3] D. Moroz, P. Nelson, R. Bar-Ziv, and E. Moses, Phys. Rev. Lett. 78, 386 (1997).

[4] D. G. Grier, J. Phys.:Cond. Mat. 12, A85 (2000).

[5] N. Maurer, D. B. Fenske, and P. R. Cullis, Expert Opin Biol TH 1, 923 (2001).

[6] Y. S. Park, Bioscience Rep. 22, 267 (2002).

[7] B. V. Deryagin and Y. V. Gutop, Colloid. J. USSR 24, 370 (1962).

[8] C. Taupin, M. Dvolaitzky, and C. Sauterey, Biochemistry 14, 4771 (1975).

[9] J. Happel and H. Brenner, Low Reynold number hydrodynamics (Martinus Nijhoff, Dordrecht, 1986).

[10] O. Sandre, L. Moreaux, and F. Brochard-Wyart, Proc. Natl. Acad. Sci. USA 96, 10591 (1999).

[11] F. Brochard-Wyart, P. G. de Gennes, and O. Sandre, Physica A 278, 32 (2000).

[12] D. V. Zhelev and D. Needham, Biochim. Biophis. Acta 1147, 89 (1993).

[13] W. Helfrich and R. Servus, Nuovo Cimento Soc. Ital. Fis.
3D, 1 (1984).

[14] E. Evans and W. Rawicz, Phys. Rev. Lett. 64, 2094 (1990).

[15] P. Sens and H. Isambert, Phys. Rev. Lett. 88, 128102 (2001).

[16] G. Debregeas, P. Martin, and F. Brochard-Wyart, Phys. Rev. Lett. 75, 3826 (1995).

[17] H. T. Tien, Bilayer Lipid Membranes (Marcel Dekker, New York, 1974).

[18] J. C. Weaver and Y. A. Chizmadzhev, Biolectrochem. Bioenerg. 41, 135 (1996).

[19] T. Steck and et al, Science 168, 255 (1970).

[20] T. Steck and J. Kant, Meth. Enzym. 31, 172 (1974).

[21] M. R. Lieber and T. Steck, J. Biol. Chem. 257, 1982 (1982).

[22] M. D. Betterton and M. P. Brenner, Phys. Rev. Lett. 82, 1598 (1999).

[23] Y. Levin, Rep. Prog. Phys. 65, 1577 (2002).

[24] J. C. M. Mombach et al., Physica A 297, 495 (2001).

[25] J. C. M. Mombach and J. A. Glazier, Phys. Rev. Lett. 76, 3032 (1996). 